SHS Web of Conferences 6, 02004 (2014)

DOI: $10.1051 /$ shsconf / 20140602004

(C) Owned by the authors, published by EDP Sciences, 2014

\title{
Research on the Identity Construction of the Disabled Elderly in Rural Areas of China
}

Chen $\mathrm{Xu}$

Department of Sociology, Wuhan University, 430072 Wuhan Hubei, China,

\begin{abstract}
The identity construction is a very important aspect among the problems of the disabled elderly in rural areas of China. Based on the method of case study and theoretical analysis, this research studies the contents and the process of the identity construction of the disabled elderly in rural areas. It suggests that the identity consists of three elements: personal identity, group identity and social identity, and the characteristics of which include the "high recognition on the disability", the "core construction of the disability", the "daily expenses orientation" and the "de-certification of the identity construction". The mechanism of the construction is supported by three different system types: the basic system, the consolidation system and the maintenance system. The results of the construction demonstrate the features of the identity of "triple-weakness" and "muddling along" of the disabled elderly in rural areas.
\end{abstract}

Keywords. disabled elderly; rural areas; identity construction; research

\section{Research background}

\subsection{Introduction}

The data of the Second National Survey on the disabled elderly shows that the elderly disabled in rural areas constitute $75.04 \%$ of the disabled in China. The total amount of the population is 31 million. Compared with the metropolises, those who live in rural areas are usually troubled with disability, low income, and the inferior status of the Medicare. The deteriorating status of the disabled rural elderly has brought many negative effects to the society, economy and culture.

The identity construction of the rural disabled elderly is an unfamiliar and yet important part of the problems of the ageing in China. In terms of the unfamiliar, there are a few related researches on this topic and in terms of the importance, the identity construction consists of many aspects, including the self-recognition on the social identity, social status and so on. It is directly linked to the mental status of the disabled elderly and it is a reflection of how the recognition of the disabled elderly is formed. The mental status is the vital factor that influences the living quality [1]. Thus, the identity construction plays a very important role in daily lives of the disabled elderly.

\subsection{The concept of the identity construction}

The identity construction means the "self" and "identity". The "self" means the recognition on someone's identity and the "identity" means the recognition on things that has the same characteristics

This is an Open Access article distributed under the terms of the Creative Commons Attribution License 2.0, which permits unrestricted use, distribution, and reproduction in any medium, provided the original work is properly cited. 
of the disabled elderly in rural areas [2]. The concept of the "identity construction" mainly discusses the following questions:

"Who am I?" This question depicts the true characteristics of the disabled elderly living in rural areas.

"What is my identity?" This identity, so to speak, contains two different aspects: the first one is that it is a microscopic character which shows the role of the disabled elderly in the family, and the second one shows the forms of the disabled elderly.

"What is my behavior mode?" The behavior mode, so to speak, is an objective concept. To the rural disabled elderly, it contains some basic activities such as taking medicine, doing rehabilitation activities, and doing some housework if their bodies function well. Also, the behavior mode means the principles of their daily lives, such as getting used to the lives after they have become disabled.

The construction consists of two meanings. One suggests the confirmation of the identity of the disabled elderly may take a lot of time and the other suggests the result of mutual factors. Thus, the confirmation of the identity of the disabled elderly in rural areas is the result of many factors.

Therefore, there are three different aspects in analyzing the identity construction of the disabled elderly in rural areas: the analysis on the process of construction; the analysis on the mechanism of construction and the result of construction, which lead to the characteristics of the identities of the rural disabled elderly.

\subsection{Research method}

This research uses both qualitative and theoretical research methods. The researcher has used indoor intensive interview method, interviewed fifteen disabled elderly people, and recorded the whole research process under the permission of the respondents.

\section{The core identities of the disabled elderly in rural areas}

The identities of the disabled elderly contribute to the main characteristics of them. They form the basis for researching on the identity construction. In fact, to every social member, the external identities may be characterized by the varieties and complexities [3]. Therefore, it is of vital importance doing researches on the core and natural identities of the rural disabled elderly.

\subsection{The personal identity of the disabled elderly in rural areas}

Disability is the key personal identity of the rural disabled elderly. On the one hand, the disability destroys the physical and mental health of the rural disabled elderly. On the other hand, it greatly affects the self-care abilities and communication skills. Thus, to the rural disabled elderly themselves, the disability itself has been integrated with their daily lives. They have to be aware that they are "disabled" all the time. However, most of the rural disabled elderly calmly accept the existence and reality of disability and take it for granted. Therefore, the integration of disability and the disabled elderly becomes the personal attribution of the rural disabled elderly.

As the disability negatively affects the self-care abilities of the disabled elderly, they tend to show a higher reliance upon the external support. Thus, they are usually "the ones being taken care of". Being different from the disabled senior citizens in metropolises, the rural disabled elderly are in lack of the choices of the resources of the care givers. Spouses, offspring, and relatives take almost all the responsibilities of caring and it makes the characteristic of "the ones being taken care of" become one of the most obvious identities of the rural disabled elderly. The formation and construction of this characteristic is deeply related to the disability itself.

\subsection{The group identity of the disabled elderly in rural areas}


The group identity of the rural disabled elderly can be categorized as two types: the "marginal men" and "the men of oblivion".

The concept of the "marginal men" demonstrates that the elderly are usually far from the center of social activities. They receive less attention and live at the "margins" of the social activities. To those who live in rural areas, they seldom take part in the agricultural activities. Therefore, not only the young peasants regard the rural disabled elderly as the "marginal men", but the rural disabled elderly themselves feel they are useless. "Just taking care of themselves" becomes the main attitude of the rural disabled elderly, and this contributes to the closed identity of "marginal men." In fact, the identity of the disabled elderly is always isolated from the perspective of groups. Disability plays a part in this process. However, the key factors are the loose structure of the rural communities, the production-orientation in rural areas' daily lives, and the delay of the old age programs that results in the identity of the "marginal men" of the rural disabled elderly nowadays.

"The men of oblivion" is a vivid description of the identity of the rural disabled elderly. The word "oblivion" means the rural disabled elderly are isolated from the rural construction, welfare system, and social security. The formation of the "marginal men" is influenced by the personal factors of the rural disabled elderly themselves while "the men of oblivion" is firmly connected to the problems of the delay of social programs for the elderly and disabled in rural areas. This research concludes as follows:

The oblivion of recognitions. It means the government officials do not realize the severity of problems of the rural disabled elderly and neglect them.

The oblivion of policies. On the one hand, it means the policy neglects the rural disabled elderly. On the other hand, it means the executors fail to practice enough care for the rural disabled elderly.

\subsection{The social identity of the disabled elderly in rural areas}

The social identity of the rural disabled elderly is generally reflected as the "Weakness" because the social support for the disabled elderly is relatively weak in rural areas. What is more, the growing of the "empty nester" demonstrates that the elderly are facing great problems of the social support.

\section{The main characteristics of the identity construction of the disabled elderly in rural areas}

To the rural disabled elderly, the identity construction is usually group-oriented, the characteristics are as follows:

The high recognition on the weakness, disability and helplessness. The disabled elderly usually treat their identities calmly and accept the negative identities such as "the disabled", "the useless" and so on.

The core construction of the disability. The term "disability" itself brings physical and mental pain to the rural disabled elderly. What is more, it strengthens the identity recognition on the disability.

The daily expenses orientation. To the most of the rural disabled elderly, the daily expenses are the most important problems they care about.

The "de-certification" of the identity construction. The rural disabled elderly are not interested in the certification of the household register, the elderly register and the disabled register. In fact, they lack the social resources to help them change the identities. Therefore, they tend to live a "muddling-along" life and they are not active in changing their living situations.

\section{The mechanism of the identity construction of the disabled elderly in rural areas}

The mechanism of the identity construction is firmly related to the three different systems.

First, the fundamental system. This system is under the supervision of the rural disabled elderly. 
They accept the identities of disability, weakness and so on. They construct their own living purposes and mechanisms around such identities.

Second, the consolidation system. Consolidation here means the identities are fixed and unchangeable. To achieve this purpose, two basic conditions are needed. The first is the influencer. The influencer usually has intimate relationship to the disabled elderly, such as spouse, brother and sister. The disabled elderly voluntarily accept the influences generated by the influencer. The second is the influence itself. It needs to be successive and based on the basic identities of disability, weakness and so on. Therefore, this research suggests that the family members push forward the identity construction of the rural disabled elderly.

The action and oral expression are two different modes of this "pushing". The action mode means that the caregiving by the spouses and offspring can help push forward the identity construction. The caregiving makes the elderly realize that they are already disabled and need the support. The oral expression mode means the identity of the rural disabled elderly are consolidated by words of the caregivers. In fact, such expressions are not always humiliating.

Third, the maintenance system. The rural areas play an important role in the maintenance system. Since it is difficult for the rural disabled elderly to contact with other villagers due to the disability while the rural areas lack the equipment for the disabled elderly to take rehabilitation activities, thus the rural areas help form the maintenance system to keep the identities of the rural disabled elderly.

\section{The results of the identity construction of the disabled elderly in rural areas}

Besides constructing the unique identities of the disabled elderly, the identity construction leads to two following results:

The "triple weakness" of the rural disabled elderly. The "triple weakness" means the weakness in rural areas, in disability and in old age. Those who live in rural areas lack the supporting resources. Those who are disabled, are negatively tagged with the "inferior ones". Those who are already in the old age are relegated to the group of "weakness".

The "muddling along" of the living attitude of the rural disabled elderly. It means that on the one hand, they yield to the disability and on the other hand, they lack specific living targets [4].Therefore, "muddling along" is a chief characteristic of the rural disabled elderly.

\section{Acknowledgment}

This essay benefits from the achievement of the two following social research programs:

"Research on the Patters of Support for the Disabled Elderly", guaranteed by the China Post Doctor Fund (2013M531726).

"Research on the Support for the Disabled Elderly in Wuhan", guaranteed by 2013 Wuhan Social Science Fund (13046).

\section{References}

1. Zhao Chunluan, The Thesis Collection of the Second National Survey of the Disabled Elderly in Beijing. Beijing: Hua Xia Press, 2008, pp.67-129.

2. Wang Ying. "An Analysis of Identification and the Establishment of Identity," Journal of Henan Normal University, vol. 35, pp. 50-53, January 2008

3. Xiang Yunhua. "Summary of the Researches of the Identity Construction. Social Science Research," Social Science Research, vol. 34, pp. 188-192, October 2009.

4. Chen $\mathrm{Xu}$. "Research on the Family Long-term Support for the Disabled Elderly-Take the Disabled Elderly in Beijing as an Example," Theory Monthly, vol.357, pp. 100-103, September 2011 